\title{
Image Filtering using Linear and Non Linear Filter for Gaussian Noise
}

\author{
Pawan Kumar \\ Patidar \\ Assistant Professor \\ Rajasthan Technical \\ University, Computer \\ Science Department, \\ Vivekananda \\ Institute of \\ Technology, \\ Rajasthan, INDIA
}

\author{
Lalit \\ Assistant Professor \\ Rajasthan Technical \\ University, Computer \\ Science Department, \\ Vivekananda \\ Institute of \\ Technology, \\ Rajasthan, INDIA
}

\author{
Baldev Singh \\ Professor \\ Computer Science \\ Department, \\ Vivekananda \\ Institute of \\ Technology (East), \\ Rajasthan, INDIA
}

\author{
Gaurav Bagaria \\ Assistant Professor \\ Computer Science \\ Department, \\ Vivekananda \\ Institute of \\ Technology, \\ Rajasthan, INDIA
}

\begin{abstract}
The goal of image filtering is to remove the noise from the image in such a way that the "original" image is visible. Linear filter [1] is the filtering in which the value of an output pixel is a linear combination of neighborhood values, which can produce blur in the image. Median filter is the one of the most popular non-linear filter. Image filtering is a method by which we can enhance images .Image filtering methods are applied on images to remove the different types of noise that are either present in the image during capturing or injected into the image during transmission. In these work Gaussian noise used and image filtering performed by Linear and Non Linear filter. Further results have been compared for filters using Standard Deviation and Mean Square Error value.
\end{abstract}

\section{Keywords}

Gaussian noise, Linear Filter, Non Linear Filter, Standard Deviation, Mean Square Error.

\section{INTRODUCTION}

Image Processing [2] is a form of signal processing for which the input is an image, such as a photograph or video frame; the output of image processing may be either an image or, a set of characteristics or parameters related to the image.
Linear filters generally blur sharp edges; destroy lines and other fine details present in the image. To overcome the problem of linear filtering, non-linear filtering [1] techniques become popular as an alternative to preserve signal structure. Wiener filter is a linear filter and median and fuzzy filter are non linear filter. In this paper; we perform wiener, median and fuzzy filtering to de-noise an image [4]. Fuzzy Filter can manage the imprecision and ambiguity in many image processing applications efficiently. Median filtering is generally superior to linear filtering (e.g., moving average filter) on effectively suppressing impulse noise. We suggest to de-noise a degraded image $X$ given by $X=S+N$, where $S$ is the original image and $\mathrm{N}$ is an Additive White Gaussian noise with unknown variance. The rest of the paper is organized as follows:-

- In the second section we review the Fuzzy Filter.

- In the third section we present the method of Median filter.

- In the fourth section we present the method of Wiener filter.

- In the fifth section we described types of noise.

- The simulation results are discussed in part six.

- We conclude and future work in part seven and eight.
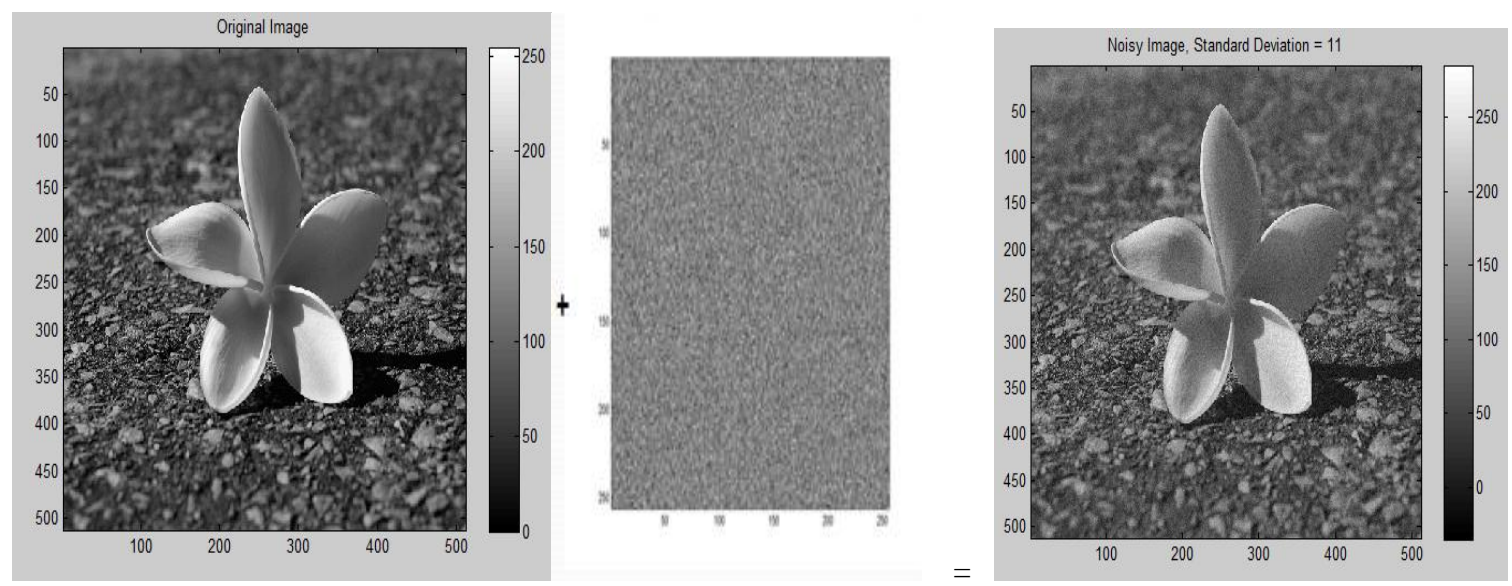

Figure 1: The original image, an Additive White Gaussian Noise, and noisy image 


\section{FUZZY FILTER}

Fuzzy Filter (FF) is based on gray level mapping into a fuzzy plane, using a membership function [5]. The aim is to generate an image of higher contrast than the original image by giving a larger weight to the gray levels that are closer to the mean gray level of the image than to those that are farther from the mean. An image $\mathrm{f}$ of size $\mathrm{M} \times \mathrm{N}$ and L gray levels can be considered as an array of fuzzy singletons, each having a value of membership denoting its degree of brightness relative to some brightness levels. For an image $f(x, y)$, we can write in the notation of fuzzy sets:

$$
\mathrm{f}(\mathrm{x}, \mathrm{y})=\underset{\mathrm{xy}}{\mathrm{U}} \mu_{\mathrm{xy}} / \mathrm{I}_{\mathrm{xy}}
$$

where

$\mathrm{x}=1,2, \ldots ., \mathrm{M}$

$\mathrm{y}=1,2, \ldots ., \mathrm{N}$

$\mathrm{I}_{\mathrm{x} y}=$ The intensity of $(\mathrm{x}, \mathrm{y})$ th value

$\mu_{\mathrm{xy}}=$ Membership value.

The membership function characterizes a suitable property of image like darkness, edginess, textural property etc. and can be defined globally for the whole image or locally for its segments. The basic principles of fuzzy enhancement scheme are illustrated in Fig. 2.1.

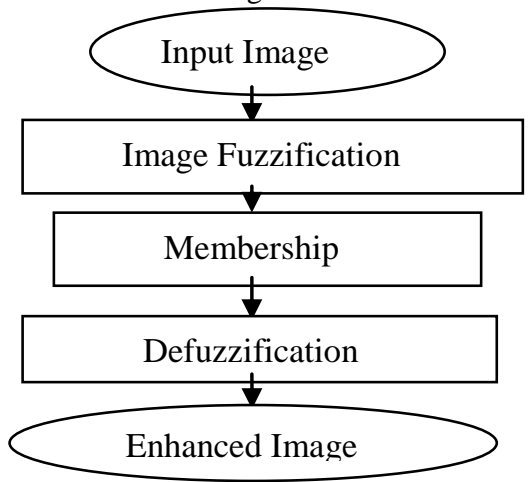

Figure 2: The basic principles of fuzzy enhancement

Fuzzy Filter method for image de-noising based on Fuzzy set theory. This filter employs Fuzzy rules for deciding the gray level of a pixel within a window in the image. This is a variation of the MF and Neighborhood Averaging filter with fuzzy values. The algorithm includes the following steps:

1. At first the gray values of the neighborhood pixels

$(n \times n$ window $)$ are stored in an array an then sorted in ascending or descending order.

2. Then, fuzzy membership value is assigned for each neighbor pixels:

This step has the following characteristics:

i. А П-shaped membership function is used.

ii. The highest and lowest gray values get the membership value 0 .

iii. Membership value 1 is assigned to the mean value of the gray levels of the Neighborhood pixels.

3. Now, we consider only $2 \times k+1$ pixels $(\mathrm{k} / 2<=\mathrm{n} 2)$ in the sorted pixels, and they are the median gray value and $k$ previous and forward gray values in the sorted list.

4.Now, the gray value that has the highest membership value will be selected and placed as output.

\section{MEDIAN FILTER}

The Median Filter (MF) is a non linear digital filtering technique. Median filtering [6] preserves edges while removing noise. The main idea of the median filter is to replacing each entry with the median of neighboring entries. Note that if the window has an odd number of entries, then the median is simple to define: it is just the middle value after all the entries in the window are sorted numerically. For an even number of entries, there is more than one possible median. Median filters are widely used as smoothers for image processing, as well as in signal processing and time series processing. The output $y$ of the median filter at the moment $t$ is calculated as the median of the input values corresponding to the moments adjacent to $\mathrm{t}$ :

$\mathrm{y}(\mathrm{t})=$ median $\left(\left(\mathrm{x}(\mathrm{t}-\mathrm{T} / 2), \quad \mathrm{x}\left(\mathrm{t}-\mathrm{T}_{1}+1\right), \ldots, \quad \mathrm{x}(\mathrm{t}), \ldots, \quad \mathrm{x}(\mathrm{t}\right.\right.$ $+\mathrm{T} / 2)) \ldots \ldots \ldots \ldots . . .(3.1)$

Where $t$ is the size of the window of the median filter. Each pixel is represented by several values, e.g. by its "red", "green", "blue" values determining the color of the pixel.

\section{WIENER FILTER}

The goal of the Wiener Filter (WF) is to filter out noise that has corrupted a signal. It is based on a statistical approach. The Wiener filter [7] approaches filtering from a different angle. Performance criteria of wiener filter is minimum meansquare error.

\subsection{Wiener Filter in the Fourier Domain}

The Wiener filter is:

$$
G(u, v)=\frac{H^{*}(u, v) P_{s}(u, v)}{|H(u, v)|^{2} P_{s}(u, v)+P_{n}(u, v)}
$$

Dividing through by $\mathbf{P}_{\mathrm{s}}$ makes its behavior easier to explain:

$$
\begin{aligned}
& \mathrm{G}(\mathrm{u}, \mathrm{v})=\quad \mathrm{H}^{*}(\mathrm{u}, \mathrm{v}) \\
& |\mathrm{H}(\mathrm{u}, \mathrm{v})|^{2}+\frac{\mathrm{P}_{\mathrm{n}}(\mathrm{u}, \mathrm{v})}{\mathrm{P}_{\mathrm{s}}(\mathrm{u}, \mathrm{v})}
\end{aligned}
$$

The term $\mathbf{P}_{\mathbf{n}} / \mathbf{P}_{\mathrm{s}}$ can be interpreted as the reciprocal of the signal-to-noise ratio.

\section{IMAGE NOISE}

Image noise [8] is the random variation of brightness or color information in images produced by the sensor and circuitry of a scanner or camera. The types of Noise are following:-

- Gaussian noise

- Salt-and-pepper noise

- $\quad$ Speckle noise

- Quantization noise

- Film grain

- Non-isotropic noise 


\section{A. Gaussian noise}

The standard model of amplifier noise is additive, Gaussian, independent at each pixel and independent of the signal intensity, caused primarily by thermal noise, including that which comes from the reset noise of capacitors ("kTC noise").Gaussian noise is a major part of the "read noise" of an image sensor, that is, of the constant noise level in dark areas of the image [8]. Gaussian noise is generated by formula given below:

$$
\text { Noise }=\mathrm{x} * \mathrm{y}
$$

Where $\mathrm{x}$ is standard deviation and $\mathrm{y}$ is randomly generated matrix of image size (M, M).Performances of different filters are tested for three different types of noise models by calculating the MSE and PSNR. The values are calculated by the following expressions:

MSE is given by

$$
M S E=\sum_{\mathrm{x}=1}^{\mathrm{M}} \sum_{\mathrm{y}=1}^{\mathrm{N}}(f(x, y)-k(x, y))^{2} .
$$

Where $\mathrm{M}, \mathrm{N}$ is total number of pixels and $\mathrm{f}$ and $\mathrm{k}$ are original and filtered image respectively.

$$
\text { PSNR }=20 \log _{10} \frac{256}{\text { Sqrt }(M S E)}
$$

Where PSNR, MSE represents the peak signal to noise ratio and mean square error of the estimation.

\section{SIMULATION RESULTS}

In this section, experimental results are presented which explored the characteristics of the various filters used and tested. The comparative analysis has been presented on the basis of different standard deviation of noise for the original image $(512 * 512)$ which is shown in the Table (1). The result is taken by comparing the performance of MF, FF, and WF on the basis of PSNR and MSE value.

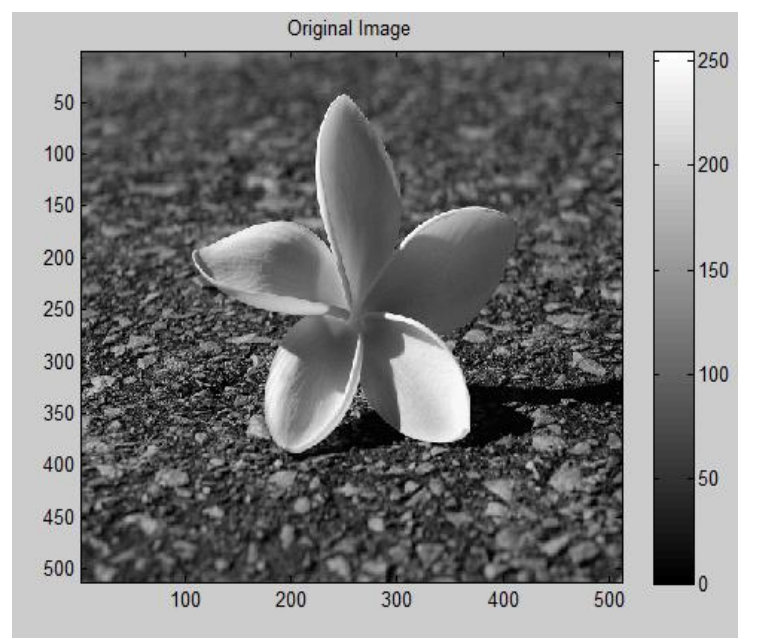

Figure 3: Original Image

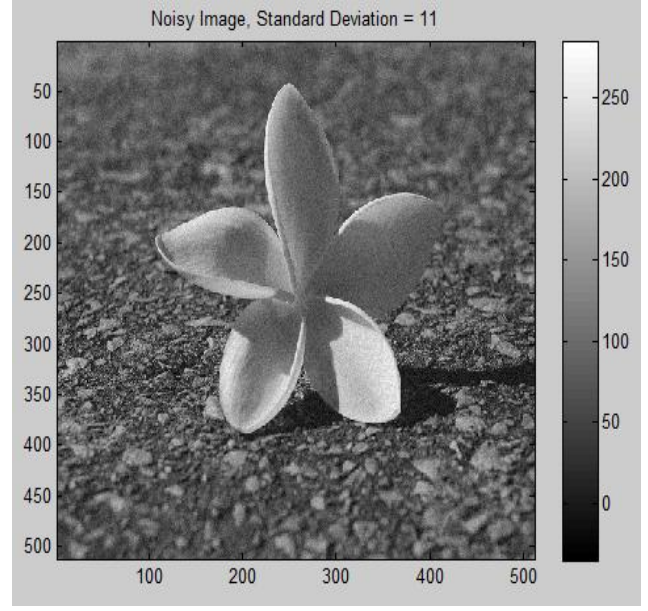

Figure 4: Adding Gaussian noise with Standard Deviation

(11)

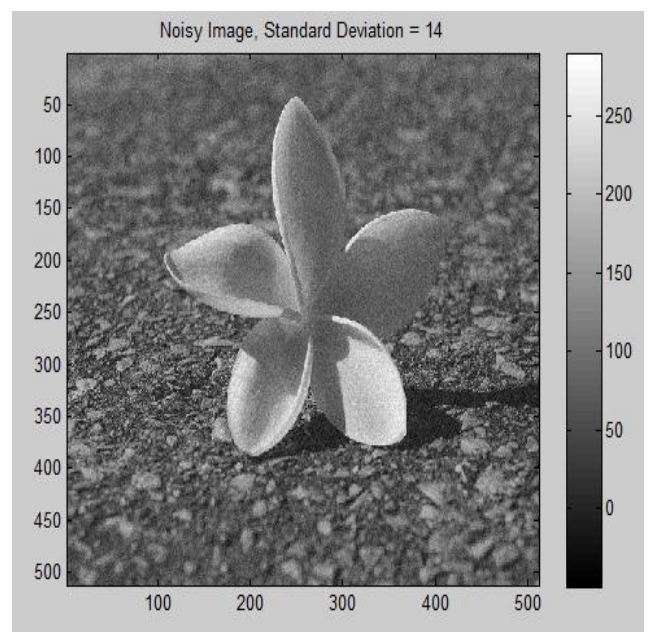

Figure 5: Adding Gaussian noise with Standard Deviation

(14)

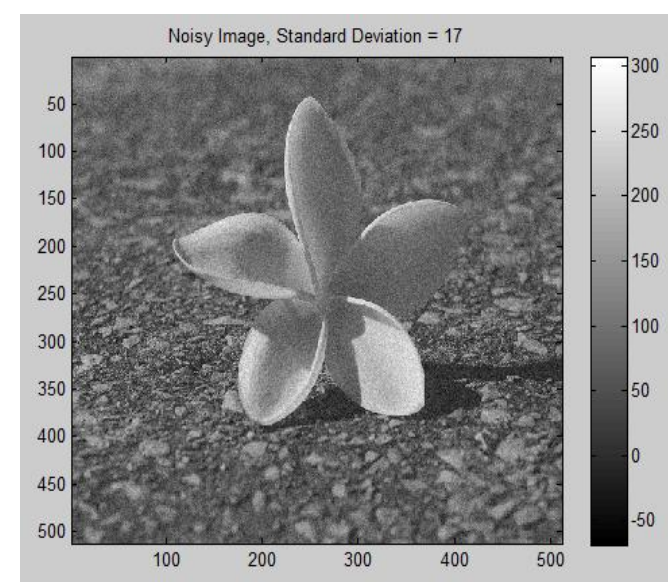

Figure 6: Adding Gaussian noise with Standard Deviation

(17) 


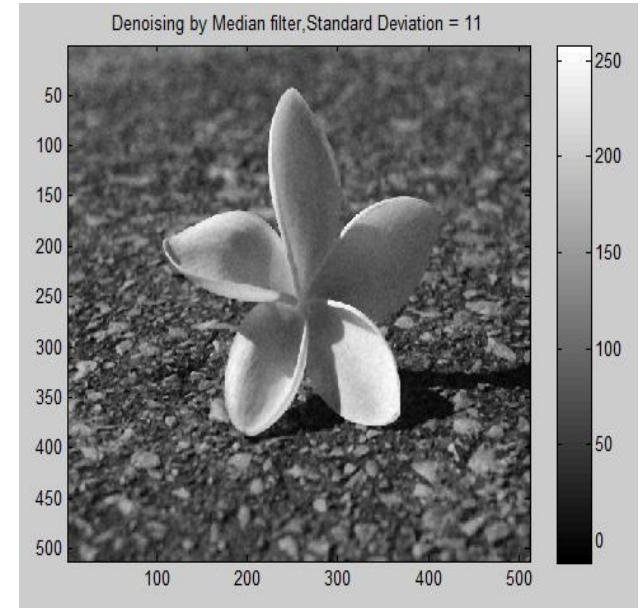

Figure 7: De-noising by Median Filter, Standard Deviation $=11$

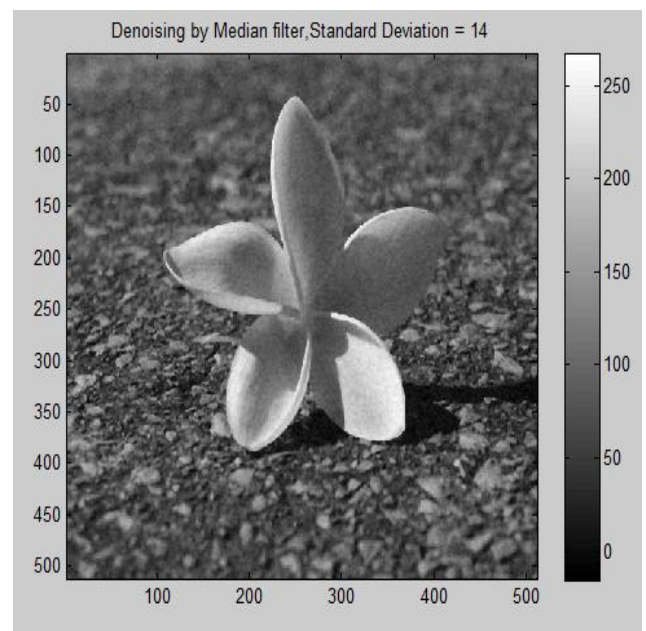

Figure 8: De-noising by Median Filter, Standard Deviation $=14$

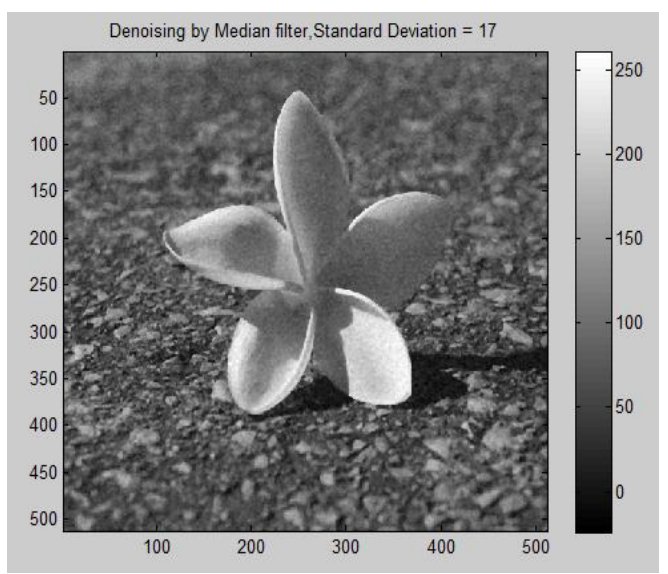

Figure 9: De-noising by Median Filter, Standard Deviation $=17$

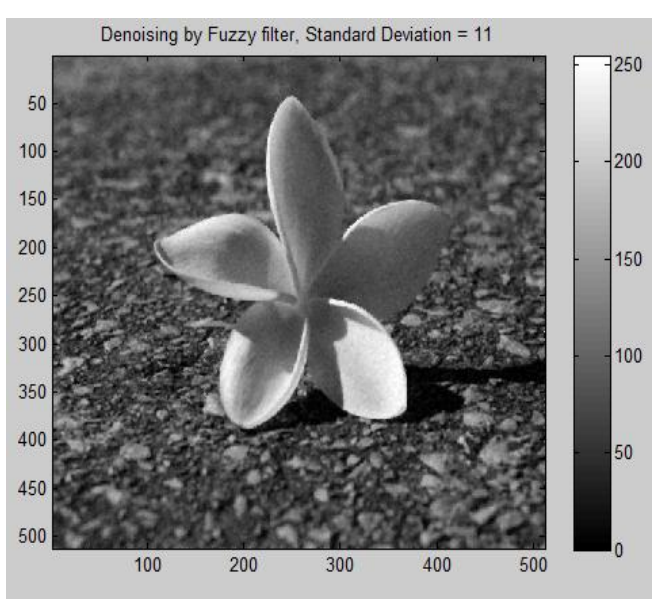

Figure 10: De-noising by Fuzzy Filter, Standard Deviation $=11$

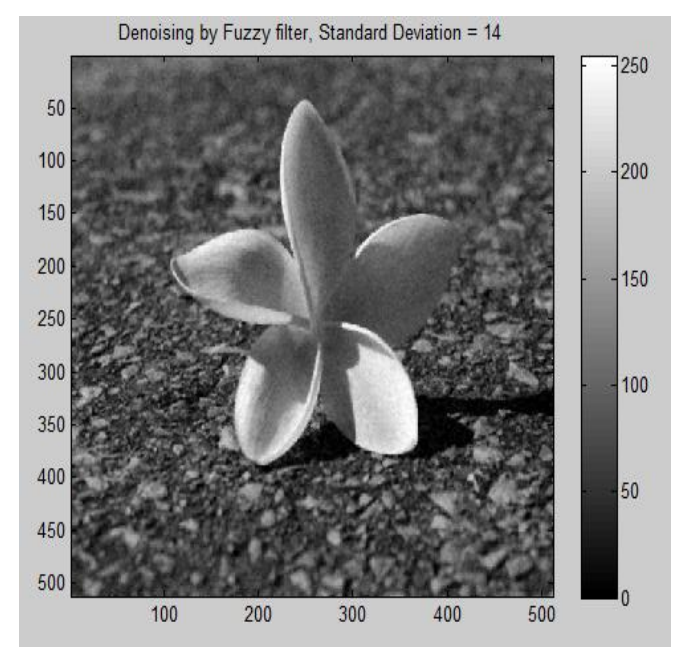

Figure 11: De-noising by Fuzzy Filter, Standard Deviation $=14$

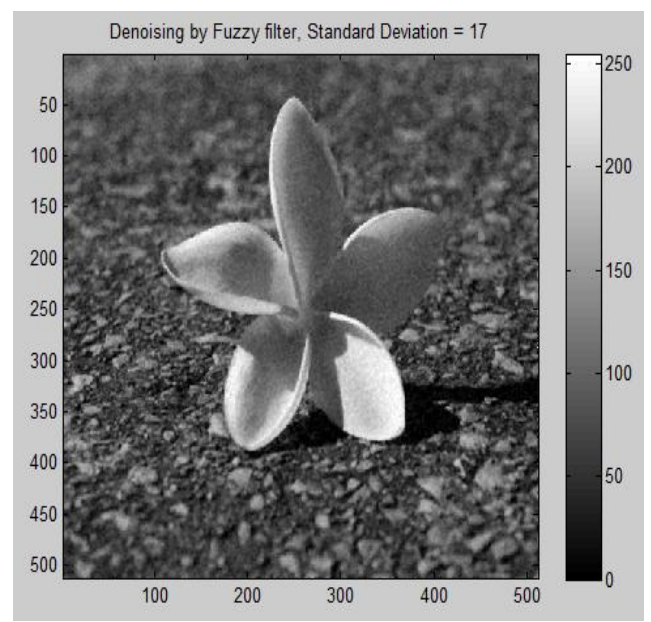

Figure 12: De-noising by Fuzzy Filter, Standard

Deviation $=17$ 


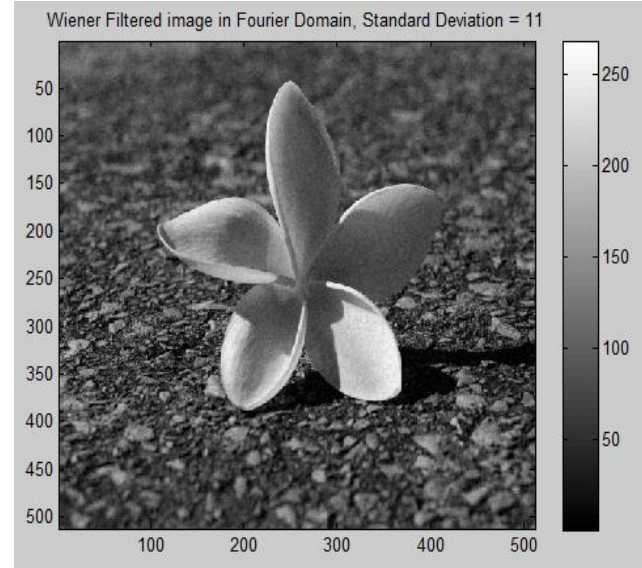

Figure 13: De-noising by Wiener Filter, Standard

\section{Deviation $=11$}

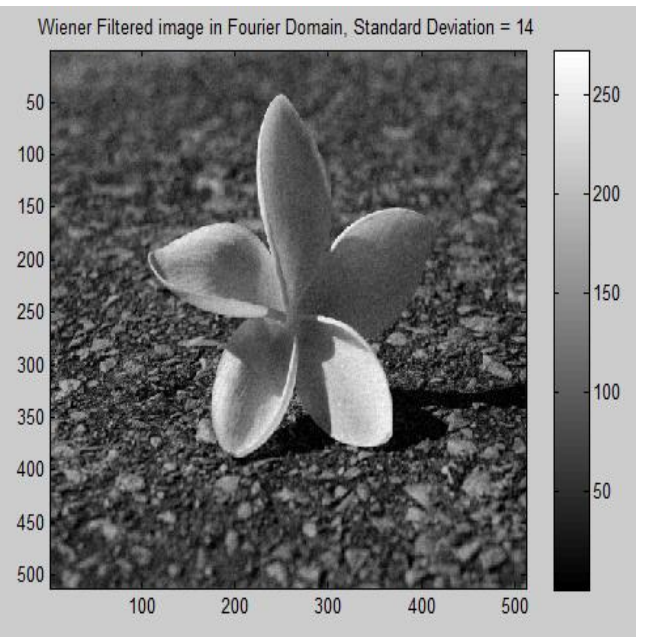

Figure 14: De-noising by Wiener Filter, Standard

Deviation $=14$

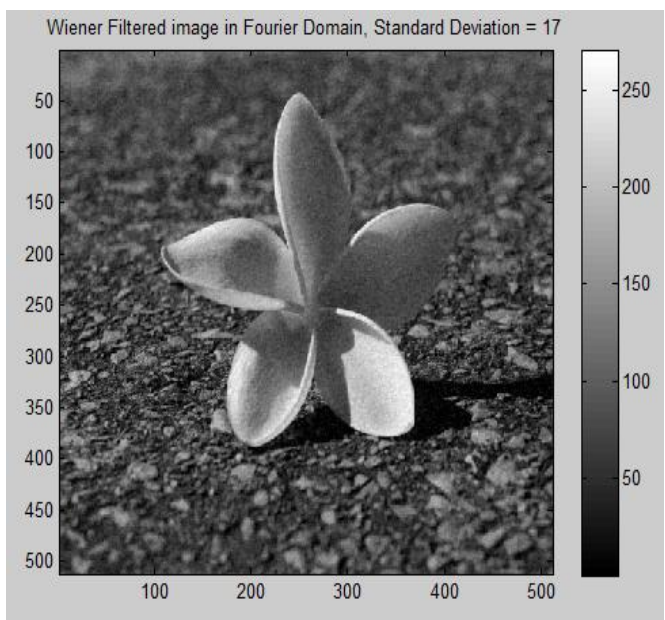

Figure 15: De-noising by Wiener Filter, Standard Deviation $=17$
Table 1. Performance Measure of Median, Wiener and Fuzzy Filter

\begin{tabular}{|c|c|c|c|}
\hline Filter & $\begin{array}{c}\text { Standard } \\
\text { Neviation } \\
(\sigma)\end{array}$ & $\begin{array}{c}\text { MSE } \\
\text { Value }\end{array}$ & $\begin{array}{c}\text { PSNR } \\
\text { Value }\end{array}$ \\
\hline \multirow{3}{*}{$\begin{array}{l}\text { Wiener } \\
\text { Filter }\end{array}$} & 11 & 58.3649 & 30.4693 \\
\cline { 2 - 4 } & 14 & 78.0919 & 29.2047 \\
\cline { 2 - 4 } & 17 & 97.9585 & 28.2204 \\
\hline \multirow{2}{*}{$\begin{array}{l}\text { Fuzzy } \\
\text { Filter }\end{array}$} & 11 & 151.2294 & 26.3344 \\
\cline { 2 - 4 } & 14 & 162.2080 & 26.0301 \\
\hline \multirow{2}{*}{$\begin{array}{l}\text { Median } \\
\text { Filter }\end{array}$} & 11 & 176.3163 & 25.6679 \\
\cline { 2 - 4 } & 17 & 174.5954 & 25.7105 \\
\cline { 2 - 4 } & 14 & 155.9727 & 26.2003 \\
\hline
\end{tabular}

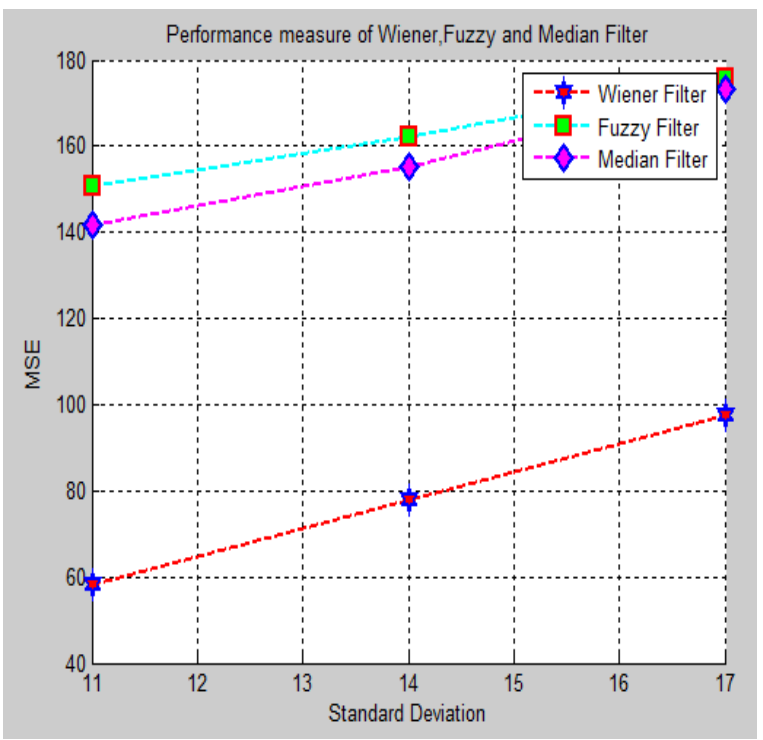

Figure 14: Performance measure of Wiener, Median and Fuzzy Filter

\section{CONCLUSION}

This paper focus on the effective algorithms which has been used for Image Filtering by using median, wiener and fuzzy filter which tell that: The performance of wiener filter is better than median filter and fuzzy filter according to MSE value from Figure 14 because of the performance of wiener filter is based on minimum mean- square error.

\section{SCOPE FOR FUTURE WORKS}

Future works include use new concepts to modification of membership value without affecting the performance of result in Fuzzy filtering. Use adaptive properties for comparison of all filters. The construction of other fuzzy filtering methods for color and gray images to filtering other types of noise (salt $\&$ pepper, quantization, speckle, etc.). 


\section{REFERENCES}

[1] T.K.Thivakaran, Dr.RM.Chandrasekaran, "Nonlinear Filter Based Image Denoising Using AMF Approach" International Journal of Computer Science and Information Security, Vol. 7, No. 2, 2010

[2] Charu Khare , Kapil Kumar Nagwanshi, "Image Restoration Technique with Non Linear Filter", International Journal of Advanced Science and Technology ,Vol. 39, February, 2012

[3] R. C. Gonzalez and R.E. Woods, Digital Image Processing, Prentice Hall, 2008.

[4] Mukesh C. Motwani, Mukesh C. Gadiya, Rakhi C. Motwani, Frederick C. Harris, Jr, (2004) "Survey of Image Denoising Techniques," Proc. of GSPx 2004, Santa Clara Convention Center, Santa Clara, CA, pp. 2730 .
[5] M. Mozammel Hoque Chowdhury, Md. Ezharul Islam, Nasima Begum and Md. Al-Amin Bhuiyan "Digital Image Enhancement with Fuzzy Rule-Based Filtering", IEEE,1-4244-1551-9/07, 2007.

[6] James C. Church, Yixin Chen, and Stephen V. Rice Department of Computer and Information Science, University of Mississippi, "A Spatial Median Filter for Noise Removal in Digital Images", IEEE, page(s): 618623, 2008.

[7] Wavelet domain image de-noising by thresholding and Wiener filtering. Kazubek, M. Signal Processing Letters, IEEE, Volume:10, Issue: 11, Nov. 2003265 Vol.3.

[8] Charles Boncelet(2005)."Image Noise Models". in Alan C. Bovik. Handbook of Image and Video Processing. 